Caocci, D. (2020). Ironia e sarcasmo in Un filo di fumo di Andrea Camilleri. Italica Wratislaviensia, 11(2), 41-54.

DOI: http://dx.doi.org/10.15804/IW.2020.11.2.3

\author{
Duilio Caocci \\ Università degli Studi di Cagliari, Italia \\ dcaocci@unica.it \\ ORCID: 0000-0001-5432-3137
}

\title{
IRONIA E SARCASMO \\ IN UN FILO DI FUMO DI ANDREA \\ CAMILLERI
}

\section{IRONY AND SARCASM IN ANDREA CAMILLERI'S UN FILO DI FUMO}

\begin{abstract}
This article examines the representational modes of the relationships in Andrea Camilleri's Vigata community and, in particular, those presented in Un filo di fumo. Our initial consideration is that only by combining a semantic perspective with a pragmatic one, the measurement of Sicilianisms and the evaluation of the quality of the Sicilian-Italian language can provide useful information. We need to analyse the relationship that exists between the extra-linguistic hints provided by the narrator and by the characters and the dialogues interspersed in the novel to realise that one needs to be wary of the literal sense of the text in order to fully grasp the significance of many statements. In other words, speakers make constant use of oblique strategies of communication, amongst which, this article argues, irony and sarcasm (in the current interpretation offered by the philosophy of language) emerge as prevailing. As irony is seldom presented as dislocated from an environmental, relational, and historical context, both the narrator and the characters are charged with the duty of providing indispensable information. The Sicilian-Vigatese world arising from some of Camilleri's novels takes on a semiotic specificity, then, that can be deciphered only with adequate linguistic and cultural competence.
\end{abstract}

Keywords: Andrea Camilleri, irony, sarcasm, pragmatics, identity 
È

certamente corretto affermare che l'atmosfera 'siciliana' dei romanzi di Andrea Camilleri si deve soprattutto alla qualità della lingua utilizzata, cioè a quella originale maniera di mescolare materia linguistica siciliana e italiana, e alla quantità di sicilianismi disseminata nei testi ${ }^{1}$. Ci sono tuttavia altri elementi di cui bisogna tener conto per spiegare quanto sia efficace la rappresentazione del contesto 'vigatese' che ci pare di riconoscere con un certo grado di precisione come siciliano ${ }^{2}$. È noto che l'autore abbia immesso una quantità crescente di lessico siciliano dai primi romanzi agli ultimi ${ }^{3}$ e che i primi editori che ebbero tra le mani i manoscritti d'esordio ${ }^{4}$ esprimessero forti dubbi sulla possibilità di immettere sul mercato libri caratterizzati da tale mistilinguismo. Ma se consideriamo con attenzione ciò che dice l'autore nella postfazione alla seconda edizione di Un filo di fumo (Sellerio, 1997), pubblicata a diciassette anni di distanza dalla prima (Garzanti, 1980), possiamo vedere che la questione della lingua si inserisce all'interno di un progetto complesso che ha l'ambizione di rappresentare un mondo intero con la sua cultura materiale, con abitudini, vizi e virtù coltivati in un tempo lungo.

Nel 1980 Livio Garzanti volle pubblicare questo mio romanzo risolvendo le perplessità di alcuni suoi eminenti collaboratori. Mi domandò però, quasi a guardarsi le spalle, un glossario. Comprendendo le sue taciute ragioni, principiai a compilarlo di malavoglia: poi, a poco a poco ci pigliai gusto e me la scialai. Il romanzo viene ora ristampato a distanza di diciassette anni

1 Sulla lingua e sullo stile di Andrea Camilleri disponiamo oggi del saggio ampio e sistematico di Matt, 2020. Si vedano anche Longhitano, 2001; Bertini Malgarini \& Vignuzzi, 2008; Arcangeli, 2004; Librandi, 2010.

2 Contribuisce alla creazione di questa 'atmosfera' siciliana anche la continua evocazione dei grandi scrittori che hanno costruito e alimentato il nostro immaginario sull'Isola: Verga, De Roberto, Pirandello, Sciascia, Bufalino, Consolo appaiono spesso nella produzione di Camilleri. Alcuni - Pirandello e Sciascia in particolare - rappresentano un punto di riferimento costante. Cf. almeno Marci, 2019; Danti, 2014; Zangrandi, 2016.

${ }^{3}$ Fanno eccezione ovviamente alcuni romanzi scritti interamente in italiano.

${ }^{4}$ Le prime redazioni del Corso delle cose sono della fine degli anni Sessanta, la prima edizione del romanzo per i tipi di Lalli è del 1978. Una ricostruzione suggestiva dello stesso autore sul difficile esordio è Camilleri, 19982; si veda anche Sulis, 2010. Sull'orientamento delle sperimentazioni nella prosa italiana di questa stagione cf. Matt, 2011. 
e il glossario, nel frattempo, è diventato superfluo. Se ora lo ripubblichiamo è perché la cosa sottilmente ci diverte.

Lo spunto di Un filo di fumo me lo diede un volantino anonimo, trovato tra le carte di mio nonno, che metteva in guardia contro i maneggi di un commerciante di zolfi disonesto. Per il resto, nomi e situazioni sono da addebitare alla mia fantasia. Allora, quando uscì, il romanzo piacque a mia madre: lo dedico alla sua memoria. (Camilleri, 1997², p. 123)

Oltre a quanto si è più spesso notato - ovvero il coraggio e la lungimiranza di Livio Garzanti che decide di investire sul libro contro l'opinione dei suoi consulenti e la consapevolezza dello scrittore di aver ormai, nel 1997, arricchito notevolmente il vocabolario dei suoi molti lettori - in questo testo colpisce il modo in cui Andrea Camilleri posiziona se stesso e la sua storia al centro della catena familiare. Nelle ultime righe del testo appena citato sono implicate tre generazioni di siciliani e una porzione di tempo che interessa la storia dello scrittore, ma anche la storia dei rapporti tra l'Isola e la Penisola, la storia della letteratura siciliana moderna e la storia dei personaggi del romanzo: la notizia che ispira Un filo di fumo riemerge come un relitto tra le carte del nonno e grazie all'inventiva di Andrea Camilleri si amplia e si complica per offrirsi alla lettura del pubblico contemporaneo, per incontrare il gradimento della madre dello scrittore, alla quale, non a caso, il libro è dedicato.Il tempo del nonno è quello dei tormentati decenni post-unitari, delle rivolte dei Fasci siciliani dei lavoratori, della violenta reazione crispina, dei capolavori di Giovanni Verga e di Federico De Roberto; quello della madre è il tempo del difficile assestamento dei siciliani in un contesto politico nuovo, della più intensa frizione tra le varietà della lingua locale e la lingua italiana, della retorica nazionalista della Grande guerra, del trionfo di Luigi Pirandello.

Il «'parlato' quotidiano» che lo scrittore afferma di trovare congeniale e spontaneo per la scrittura, «quel misto di dialetto e di lingua» (Camilleri, $1998^{2}$, p. 142) su cui Camilleri decide di esercitarsi si consolida proprio nella cronologia del nonno e dei genitori, come esito di processi storico-culturali di ampia portata e di fortissimo impatto. Ed è proprio su questa 'sicilianità' in movimento, in relazione con l'italiano e con le altre lingue regionali - non sarà un caso che il rimando sia al 'parlato' domestico - che pare insistere spesso lo scrittore. 
Nelle prime pagine del romanzo di cui stiamo scrivendo già si accumulano parole e espressioni dialettali, ma è subito chiaro che quella mescola precisa di parole, per essere compresa e accettabile ha bisogno di essere riportata allo sfondo sociologico, storico e antropologico in cui i personaggi agiscono.

L'intera storia si svolge in un unico luogo, in un solo giorno, il 18 settembre $1890^{5}$, come per simulare lo schema della tragedia antica, per portare al massimo grado di intensità l'attesa dei personaggi e del lettore e per sottolineare la portata della comunicazione non verbale e soprattutto quella cinesica - nello svolgimento delle relazioni. Sarà un continuo susseguirsi di inganni e insidie, nella cui rappresentazione l'ironia gioca un ruolo fondamentale.

Nella prima scena Nené, figlio del protagonista Totò Romeres, soprannominato Barbabianca, percorre l'intera Vigàta, da un magazzino all'altro, alla ricerca di cinquemila cantàra di zolfo. Suo padre, imprenditore spregiudicato, si era venduto l'intera partita di zolfo che la ditta Jung gli aveva lasciato in deposito affinché la consegnasse al comandante della nave russa Tomorov. Ora la nave sta raggiungendo il porto e i Barbabianca rischiano la catastrofe. I Barbabianca hanno bisogno di quella consistente quantità di zolfo, ma la risposta dei magazzinieri è sempre negativa.

Il lettore apprende immediatamente che la ricerca di Nené è del tutto vana e che i notabili di Vigàta si sono accordati per causare la rovina di questa famiglia di oscura origine e mai integrata. C'è un preciso e precoce momento in cui lo stesso Nené rivela ciò che ha compreso fin dal primo incontro e cioè che il senso letterale delle parole dei suoi interlocutori è e sarà sempre falso. Egli perciò può avvisare il lettore che «tanto la risposta dei magazzinieri la sa già e sa magari il commento pensato che non viene detto ma che però indovina lo stesso da uno sparlucchìo degli occhi, da una piega che spunta vicino al labbro... $\nu^{6}$.

5 Le ultime pagine sono dedicate alle funzioni funebri celebrate la mattina del giorno dopo, il 19 settembre, da Padre Cannata nell'antica chiesetta dei pescatori e da Padre Imbornone nella centrale Chiesa matrice.

6 Camilleri, 1998, p. 13. «L'intento ironico è [...] caratterizzato da particolari segnali facciali, tra cui, ad esempio, l'incurvamento delle sopracciglia (verso il basso 
È abbastanza chiaro fin dal principio che il narratore e i personaggi vogliono lasciarci intendere che in tale contesto comprendere significa non solo ascoltare o leggere, ma anche osservare e interpretare ciò che accade, avendo presente la storia delle relazioni che legano gli attori di una vicenda. Come potrebbe infatti Nené intuire il senso antifrastico delle risposte dei colleghi e addirittura leggere il loro pensiero, se non ricorrendo alla sua competenza in materia di linguaggio del corpo e a tutta la pregressa sua esperienza di vita in quel contesto?

Nel glossario del Gioco della mosca (Camilleri, 1995, pp. 102-104) Camilleri alla voce 'taliari' spiega che i siciliani sarebbero capaci di intendersi con precisione anche tramite un rapido colpo d'occhi, «senza aprir bocca» e che avrebbero nel tempo sviluppato questa attitudine per difendersi dai molti dominatori dell'Isola. Secondo lo scrittore, i suoi conterranei sono consapevoli «di non potersi fidare nemmeno delle parole» e perciò si servono volentieri e con destrezza dell'arte di 'taliarsi', cioè di condurre «discorsi complicati» senza aprir bocca.

Il sospetto della combutta dei notabili si fa certezza solo quando la mimica facciale ridefinisce il senso esatto delle parole. Lo scrittore, forse consapevole del disagio e della confusione del lettore non siciliano dinanzi a una comunicazione spesso opaca, arriva sino al punto di introdurre un personaggio il cui compito principale è quello di fare 'per nostro conto' esperienze di analisi di conversazioni costruite secondo le semiotiche della comunità vigatese. Si tratta di un ingegnere torinese, tale Lemonnier, sapientissimo in materia di miniere di zolfo, ma non ancora inserito nella vita cittadina.

Un giorno, mentre si trova a Palermo, gli capita di assistere a una conversazione durante la quale un signore molto semplicemente chiede al commendator Madonìa, suo compagno di viaggio: «Scusi, mi sa dire come sta il Papa?». Ciò che lo lascia esterrefatto è la furiosa, imprevedibile, repentina reazione del commendatore: «Ma non mi spacchi

o verso l'alto), sorridere, o ancora strizzare e/o roteare gli occhi, fare un cenno del capo, o quella che è indicata come 'faccia bianca', cioè assumere un'espressione del volto priva di emozione o di moto» (Scianna, 2018, p. 189). Sul dibattito dei filosofi del linguaggio intorno alla natura dell'ironia si veda Gentile (2012). 
la minchia!» (Camilleri, 1997, p. 21). Una plausibile spiegazione dell'evento gli verrà fornita il giorno dopo da un paesano ${ }^{7}$, il quale racconta che «il commendator Madonìa era un fervente papalino il quale dal Non expedit in poi si rifiutava di votare e che quindi il signore di Palermo doveva in qualche modo conoscerlo» (Camilleri, 1997, p. 21). È la voce di un narratore che seleziona queste vicende, che racconta il percorso conoscitivo e di integrazione dell'ingegnere piemontese con piglio antropologico. Lemonnier, come molti lettori, deve essere informato sul quadro all'interno del quale si svolge la trama e soprattutto sulle modalità attraverso le quali si palesa il conflitto tra le parti e, in qualità di portatore di un punto di vista estraneo, agisce all'interno del romanzo come catalizzatore di informazioni e all'esterno del romanzo - cioè in direzione del lettore non-vigatese - da informatore capace di superare l'ingannevole soglia letterale del testo. Egli, grazie all'esperienza, ha «imparato a capire qualche cosa dei siciliani» e cioè che «non erano le parole che dicevano, non erano i gesti che facevano [...]: bisognava invece stare attenti a come facevano quei gesti» (Camilleri, 1997, p. 20). Ma, proprio perché ancora non sa bene come leggere i segni e non conosce tutte le implicazioni complesse della scacchiera delle relazioni, deve giovarsi spesso di 'guide' che lo aiutino a ricostruire i fatti. E come suo informatore - e quindi indirettamente anche nostro - agisce appunto quel 'paesano', suo oscuro omologo vigatese, che svela «quale carica di ironia, anzi di feroce sarcasmo, c'era in quella che a lui era parsa un'innocentissima richiesta d'informazione» (Camilleri, 1997, p. 21).

È ovvio che nei vari passaggi delle informazioni indispensabili c'è un dislivello notevole tra quanto il torinese può imparare da altri personaggi e quanto il lettore può imparare da un narratore sapiente e letteratissimo che prima dissemina - facendosi voce ventriloqua dell'in-

7 Questo paesano ha una funzione simile a quella del contadino che spiega a Tognin, carabiniere veneziano perché il cadavere che hanno davanti ha «le gambe dentro a un sacco legato alla vita, le mani serrate dietro la schiena da una sottile cordicella» e «le scarpe consunte [...] assistimate sul petto», Camilleri, 1998, pp. 14-15. Cf. Caocci, 2020, p. 9. 
gegnere - notizie sulla salute precaria di Leone $\mathrm{XIII}^{8}$ e poi presenta un'ineccepibile analisi retorica del conflitto tra il signore palermitano e il commendator Madonìa.

In questo caso - ce lo dice il narratore riferendosi però alla presa di coscienza dell'ingegnere - abbiamo a che fare con una modalità della comunicazione, il sarcasmo, che sarebbe risultata inefficace senza quelle informazioni di carattere storico e relazionale e senza l'ulteriore specificazione di carattere performativo che introduce il dialogo tra $i$ due: «un signore ben vestito, di una certa età, si era avvicinato cerimoniosamente al commendatore e, evidentemente timoroso di disturbare, aveva domandato a voce piana: Scusi, mi sa dire come sta il Papa?» ${ }^{9}$.

Tale descrizione deve essere necessariamente attribuita al punto di vista di Lemonnier, osservatore ancora ignaro del meccanismo sottile sulla base del quale gli interlocutori entrano in contatto, e può essere valutata dal lettore come ironica solo a posteriori, quando apparirà chiaro anzitutto dalla reazione stizzita di Madonìa e poi dalla prolusione didascalica della voce narrante che, in effetti, egli ha proprio l'intenzione di disturbare 1'altro, che il suo approccio è 'cerimonioso', che la voce è 'piana' non perché l'uomo è garbato e mite, ma perché vuole enfatizzare l'impatto della stilettata sarcastica sul suo avversario. È evidente che questo evento è di quel tipo che «non ha nulla di ironico e triste in sé, ma diventa ironico sotto una certa descrizione» (Gentile, 2012 2 , p. 81).

All'ironia e al sarcasmo, oltre a soluzioni sintattiche e lessicali, sono associati segni pertinenti come l'intonazione della voce e la velocità dell'eloquio.

Seppur non vi sia una particolare intonazione ironica per sé e le caratteristiche acustiche siano influenzate dalla lingua del parlante, l'intonazione

8 «Da qualche tempo il giornale «La voce dell'Isola» stampava notizie non troppo allegre circa la salute di Papa Leone XIII, stremato - dicevano i giornalisti - dall'aver portato a compimento l'enciclica Immortale Dei sulla costituzione cristiana degli stati, e dall'averne iniziata un'altra Christianorum, nientedimeno che sull'emancipazione degli schiavi» (Camilleri, 1997, p. 20).

9 L'intero episodio su cui stiamo indugiando si trova in Camilleri, 1997, pp. 20-22. 
di un'espressione ironica è solitamente più alta o più bassa di quanto ci si aspetti. Possono essere indicativi di ironia, un rallentamento o delle pause lunghe nel parlato. (Scianna, 2018, p. 189)

Non basta, dunque, tenere conto del significato ambiguo della parola e del cortocircuito prodotto dalla frizione delle parole dette con le evidenze contestuali: occorre valutare anche fatti che la scrittura può riportare solo in modo indiretto - il comportamento, l'esecuzione orale degli enunciati, l'espressione del volto - e domandarsi perché dalle prospettive del personaggio e del lettore intelligendum est contrarium quod dicitur.

Sono semplicemente affermazioni false quelle dei magazzinieri che affermano di non possedere quella quantità di zolfo che invece possiedono, o possiamo considerarle - come abbiamo fatto sulla base degli indizi performativi forniti da Nené: lo «sparlucchìo dell'occhio» e la «piega che spunta vicino al labbro» - affermazioni ironiche? E inoltre, affinché siano riconoscibili come enunciati ironici da tutti gli attori in causa, di quali e quanti elementi pertinenti disponiamo?

Vediamo ora ordinatamente - servendoci di lettere minuscole dell'alfabeto - come Nené nel suo rocambolesco e inutile calvario di ricerca introduce alcuni dei magazzinieri con le loro risposte. Il primo della rassegna è Ignazio Xerri, un uomo «tutto zucchero e miele e naturalmente fàvuso, che uno lo capisce da come si ingiarma a guardarsi la punta delle scarpe», che articola la sua risposta così: a) «Sul serio, sono mortificato, ma ho i magazzini vacanti. Al suo posto, provare per provare, farei un salto da Michele Navarrìa». Quest'ultimo, «incazzato com'era sempre per niente», fornisce una risposta meno elaborata, più secca: b) «No, manco un grammo ch'è un grammo, di sùlfaro. Ho i magazzini sciutti all'osso» (Camilleri, 1997, p. 11).

Di grande interesse ai fini del nostro lavoro sono il discorso piuttosto articolato di un anonimo addetto e quello chiaro e distinto di don Saverio Fede, nemico giurato dei Barbabianca. Il primo, ossequioso ed enfatico, si rivolge a Nené in questo modo:

c) Spiacentissimo, don Nené, lei non immagina con quanto piacere e devozione l'avrei favorita. Ma per disgrazia proprio ieri abbiamo dovuto 
fare un carico completo e il poco che c'è rimasto è venuto a prenderselo Pasqualino Patti. Anzi, ora che ci penso, perché non prova da Patti? (Camilleri, 1997, p. 12)

Il secondo sfugge al controllo di Nené perché incastonato in una scena che vede coinvolti solo i nemici di Totò Barbabianca e si svolge a casa di don Saverio:

d) Non c'è bisogno che nessuno mi preghi. Diglielo, che io ai Barbabianca li voglio sapere cancellati dalla faccia della terra. Nei miei magazzini sùlfaro non ce n'è per gente che non sa campare. Salutami don Ciccio. (ibid., p. 15)

Anzitutto andrà notata la grande competenza sociale e retorica di Nené, il quale definisce le persone sulla base di osservazioni comportamentali di lunga durata. Egli sa, per esempio, che Xerri è «naturalmente fàvuso», che Navarrìa è sempre scontroso e per questa ragione prevede non solo il contenuto, ma anche la forma delle risposte. Questi giudizi minano la credibilità del significato letterale degli enunciati e, poiché insistono su elementi comportamentali e psicologici, stabiliscono la complementarietà del piano comunicativo e di quello espressivo al fine di orientare il lettore alla ricerca di un senso traslato. Sarà facile notare inoltre che l'anticipazione del profilo del primo e del secondo magazziniere contiene anche indicazioni di ordine testuale: se Nené osserva che Ignazio Xerri è «tutto zucchero e miele» e associa questa caratteristica alla falsità dell'uomo, allora sta anticipando una serie di aspetti strutturali, linguistici e retorici dei discorsi che seguiranno, soprattutto a) e c). Troppo «zucchero e miele», cioè un linguaggio sostenuto, enfatico, affettato, fitto di iperboli, estremamente ossequioso sono infatti spie tipiche degli enunciati ironici. In a) l'interlocutore si dichiara, non semplicemente 'dispiaciuto' di non poter assecondare la richiesta, ma addirittura 'mortificato', in b) l'anonimo inizia con un superlativo cerimonioso, spiacentissmo, e afferma che se avesse potuto, avrebbe 'favorito' Nené con 'piacere e devozione' e continua con grande reverenza il suo discorso del tutto privo di regionalismi. 
L'unico caso in cui siamo sicuri di poter intendere il senso delle affermazioni letteralmente è quello di d), che infatti non ha nulla di ironico e dice esplicitamente ciò che gli altri dicono indirettamente. Dal coro dei 'no', dal modo cioè in cui sono costruiti, con la destrezza acquisita, apprendiamo inoltre che il piano dei notabili prevede anche un versante ludico. In forza dell'ambiguità del significante e del margine di incertezza che comporta, $i$ concorrenti di Totò Barbabianca, in attesa della nave e del definitivo trionfo, intendono prendersi la soddisfazione di vedere Nené che corre disperato dall'uno all'altro per ricevere un unico, ma studiatamente multiforme, 'no': a) lo manda dall'iracondo Navarrìa, c) gli suggerisce di rivolgersi a Pasquale Patti.

La presenza di molta materia linguistica vigatese all'interno di questo e di altri romanzi di Camilleri risponde, anche per le ragioni che abbiamo finora portato, a una strategia narrativa, attraverso la quale lo scrittore pretende di raccontare un mondo che si definisce a partire dal modo in cui comunica mescolando segni linguistici e non linguistici con informazioni di varia natura sul contesto. La forza identitaria di alcuni personaggi deriva cioè dalla speciale maniera in cui ciascuno si serve delle possibilità offerte dal campo semiotico. Chi non ha accesso alle chiavi del codice è destinato a soccombere. Viene in mente l'assurda condizione in cui si trova, nella Mossa del cavallo (Camilleri, 1999), il ragionier Giovanni Bovara, siciliano cresciuto a Genova, quando viene inviato a Vigàta in qualità di Ispettore dei Mulini. Egli viene percepito come un estraneo non solo perché parla il ligure e perché è un esponente dell'amministrazione italiana, ma anche e soprattutto perché si comporta - da ogni punto di vista - come un estraneo che non capisce e non vuole capire il linguaggio corrente dei vigatesi. Scoprirà presto che per sfuggire alla ragnatela di inganni orditi contro di lui, gli converrà riacquisire l'habitus del siciliano, riprendere cioè a parlare e persino a pensare nella lingua locale, imparare a 'comportarsi' come i suoi avversari.

Un filo di fumo, non a caso, si chiude con l'episodio tragico del naufragio, favorito dalla scarsa volontà di intendere di un farista e del capitano della pilotina di soccorso. Il comandante della nave Tomorov, intento a governare la sua imbarcazione nel mare in tempesta di- 
mentica di esporre il segnale convenzionale di richiesta di soccorso (o non sa di doverlo fare) e il capitano Caci, pur vedendo chiaramente l'estremo pericolo, decide di non intervenire. Il difetto comunicativo, anzi l'incertezza generata dall'assenza di un segno chiaro, porta il capitano Caci a sospettare che il comandante intenda ingannarlo, mettendosi nella condizione di sostenere - una volta in salvo - di non dovere nulla ai soccorritori proprio per non aver avanzato nessuna richiesta esplicita.

A questo mondo insidioso, popolato da impostori abituati a usare ogni strumento pur di raggiungere i propri obiettivi deve appartenere anche l'autore - quell'autore che, come si diceva, inserisce nel racconto personaggi che aiutino il lettore a destreggiarsi nell'opacità del linguaggio e un narratore prodigo di informazioni - il quale, quando può operare in proprio, senza deleghe, agisce direttamente sul lettore per illuderlo sugli esiti estremi del racconto. Subito dopo il titolo e in diretta connessione con esso, c'è infatti una minuscola epigrafe che serve a introdurci alla lettura: ... un bel dì vedremo / levarsi un fil di fumo sull'estremo / confin del mare. / E poi la nave appare...

Si tratta di una citazione ricavata dalla prima parte del II atto della Madama Butterfly di Illica e Giacosa, un'aria eseguita dalla protagonista sulla struggente musica di Giacomo Puccini, tanto familiare all'orecchio e all'immaginario di molti, da suscitare importanti attese riguardo al momento della comparsa all'orizzonte del filo di fumo che tutti attendono. La posizione liminare, la notorietà dell'intera trama dell'opera attribuiscono a questo breve richiamo un valore prolettico rispetto all'evoluzione del romanzo. L'intenso canto di Cho-Cho-San, ancora ignara degli esiti tragici del suo amore e in attesa del ritorno di Pinkerton, ci fa credere che la Tomorov arriverà a Vigàta per scatenare conseguenze tragiche e ingiuste. Si tratta però del primo caso di deceptio - così lo definisce Bruno Porcelli (1998) - fra tanti che incontreremo. La citazione - almeno nella misura in cui ci avverte che alcuni di quelli che attendono il filo di fumo nell'imboccatura del porto resteranno delusi - anticipa in effetti un finale tragico, ma non del tipo annunciato. La nave del romanzo non arriverà mai a destinazione perché come abbiamo detto - fa naufragio. L'amore inoltre non è tra i temi che 
muovono gli eventi del romanzo, se non per l'incidentale e «boccaccesca vicenda della signora Helke, nuora del protagonista» che si accoppia clandestinamente con Totuzzo, figlio della cameriera di Totò Barbabianca (Porcelli, 1998, p. 99). L'azione ipertestuale di cui è responsabile l'autore serve da un lato a trasferire il vizio principale di Pinkerton, ovvero il cinismo del fedifrago, sul protagonista del romanzo, Totò Barbabianca, e sull'intera comunità vigatese $\mathrm{e}$, da un altro lato, a depistare le attese dei lettori.

La sua funzione più importante è in effetti quella di mostrare l'adesione dell'autore al codice che governa le relazioni sociali nel mondo immaginario di Vigàta, un codice oneroso da praticare e da apprendere, fondato su strategie comunicative tanto opache e infide.

\section{BIBLIOGRAFIA}

Camilleri, A. (1978). Il corso delle cose. Siena: Lalli.

Camilleri, A. (1980). Un filo di fumo. Milano: Garzanti.

Camilleri, A. (1995). Il gioco della mosca. Palermo: Sellerio.

Camilleri, A. (1997). Un filo di fumo. Palermo: Sellerio.

Camilleri, A. (1997)2. Glossario. In id., Un filo di fumo (p. 123). Palermo: Sellerio.

Camilleri, A. (1998). Il corso delle cose. Palermo: Sellerio.

Camilleri, A. (1998)2 ${ }^{2}$ Le mani avanti. In id., Il corso delle cose (pp. 139-145). Palermo: Sellerio.

Camilleri, A. (1999). La mossa del cavallo. Palermo: Sellerio.

Arcangeli, M. (2004). Andrea Camilleri tra espressivismo giocoso e sicilianità straniata. Il ciclo di Montalbano. In G. Marci (Ed.), Lingua, storia, gioco e moralità nel mondo di Andrea Camilleri (pp. 203-232). Cagliari: Cuec.

Bertini Malgarini, P., \& Vignuzzi, U. (2008). Capitoli per una storia linguistica del giallo all'italiana. RID, Rivista Italiana di Dialettologia, 32, 185-207.

Caocci, D. (2020). Premessa. In Quaderni camilleriani 12. Parole, musica (e immagini) (pp. 7-13). Cagliari: Grafiche Ghiani.

Danti, L. (2014). «Una vistosa omissione» o quasi. Pirandello nel Birraio di Preston di Camilleri. Italianistica, 43/3, 115-124.

Gentile, F. P. (2012). Teorie dell'ironia. APhEx, 6, 1-32. 
Gentile, F. P. (2012)². Ironia. $A P h E x, 6,74-99$.

Librandi, R. (2010). Nuovi plurilinguismi nella narrativa meridionale? In P. Del Puente (Ed.), Dialetti: per parlare e parlarne, Atti del I Convegno internazionale di Dialettologia (Potenza-Matera, 30-31 ottobre 2008) (pp. 77-92). Potenza: Ermes.

Longhitano, S. (2001). Parole proprie e parole di altri: la lingua di Andrea Camilleri. In M. Lamberti, \& F. Bizzoni (Eds.), La Italia del Siglo XX. IV jornadas internacionales de estudios italianos (pp. 327-345). Facultad de Filosofia y Letras - Universidad Nacional Autonoma de Mexico.

Marci, G. (2019). Isole mediterranee nell'immaginario narrativo di Sciascia, Camilleri e Bufalino (con un cenno a Tomasi di Lampedusa). In M. E. Ruggerini, V. Szőke, \& M. Deriu (Eds.), Isole settentrionali, isole mediterranee. Letteratura e società (pp. 257-310). Milano: Prometheus.

Matt, L. (2011). La narrativa del Novecento. Bologna: Il Mulino.

Matt, L. (2020). Lingua e stile della narrativa Camilleriana, In D. Caocci, G. Marci, \& M. E. Ruggerini (Eds.), Quaderni Camilleriani 12. Parole, musica (e immagini) (pp. 39-93). Cagliari: Grafiche Ghiani.

Porcelli, B. (1998). Un filo di fumo. Romanzo siciliano di Andrea Camilleri. Italianistica: Rivista di letteratura italiana, 27/1, 99-103.

Scianna, C. (2018). 'Lui ha detto che'. Un approccio filosofico alla pratica del discorso indiretto sarcastico. Rivista italiana di Filosofia del linguaggio, Special Issue: Italian Society of Philosophy of Language, 187-201.

Sulis, G. (2010). Alle radici dell'idioletto camilleriano. Sulle varianti de Il corso delle cose $(1978,1998)$. Letterature Straniere \&, 12, 249-278.

Zangrandi, S. (2016). L'ironia intertestuale in Il birraio di Preston di Andrea Camilleri. In A.M. Morace, \& A. Giannanti (Eds.), Letteratura della letteratura (pp. 1189-1202). Pisa: ETS.

Riassunto: L'articolo svolge alcune considerazioni intorno ai modi di rappresentazione delle relazioni all'interno della comunità vigatese nei romanzi di Andrea Camilleri e più in particolare in Un filo di fumo. L'osservazione da cui partiamo è che la misurazione della quantità di sicilianismi e la valutazione della qualità della lingua siculo-italiana possa fornire dati utili solo se si combina la prospettiva semantica con quella pragmatica. Analizziamo perciò il rapporto che intercorre tra le indicazioni extralinguistiche fornite dal narratore e dai personaggi e i dialoghi disseminati nel romanzo per notare che per accedere al senso di molti enunciati occorre sospettare della lettera del testo. I parlanti fanno continuamente ricorso a strategie discorsive oblique, tra le quali l'ironia e il sarcasmo - per come vengono intesi nei più recenti studi di filosofia del linguaggio - risultano prevalenti. Per questa ragione - ovvero perché l'ironia non si dà quasi mai con un significante isolabile da un contesto ambientale, relazionale e storico - narratore e personaggi del romanzo hanno 
il compito di elargire al lettore informazioni indispensabili. Il mondo siciliano-vigatese che emerge da certi romanzi di Andrea Camilleri si caratterizza per una semiotica specifica, che può essere decifrata solo se si possiedono competenze linguistiche e culturali adeguate.

Parole chiave: Andrea Camilleri, ironia, sarcasmo, pragmatica, identità 